\title{
The Therapeutic Value of Volumetric-Modulated Arc Therapy In Advanced Non-Small Cell Lung Cancer Primary Tumors: A Multicenter Retrospective Analysis Based On Propensity Score Matching And Comparison With Intensity-Modulated Radiotherapy
}

Jie Liu

Guizhou Medical University https://orcid.org/0000-0003-0926-5470

Tao Li

Sichuan Cancer Hospital and Institute

Wang Xiaohu

Gansu Provincial Cancer Hospital

Shengfa Su

The Affiliated Hospital of Guizhou Medical University

Qingsong Li

The Affiliated Hospital of Guizhou Medical University

Yichao Geng

The Affiliated Hospital of Guizhou Medical University

Wengang Yang

The Affiliated Hospital of Guizhou Medical University

Xiaxia Chen

The Affiliated Hospital of Guizhou Medical University

\section{Weiwei Ouyang}

The Affiliated Hospital of Guizhou Medical University

\section{Wei Zhang}

The Affiliated Hospital of Guizhou Medical UniversityThe Affiliated Hospital of Guizhou Medical University

Bing Lu ( $\square$ lbgymaaaa@163.com )

The Affiliated Hospital of Guizhou Medical University

\section{Research}

Keywords: Non-small-cell lung cancer/advanced, radiotherapy/VMAT, recent results/local control rate, survival/local progression-free survival, radiation injury/dose-volume parameter.

Posted Date: November 17th, 2021

DOI: https://doi.org/10.21203/rs.3.rs-1071095/v1 
License: (1) This work is licensed under a Creative Commons Attribution 4.0 International License. Read Full License 


\section{Abstract}

Objective: To explore the feasibility of volumetric-modulated arc therapy (VMAT) instead of intensity-modulated radiotherapy (IMRT) for primary tumors of advanced non-small-cell lung cancer (A-NSCLC).

Methods:.We used propensity score matching (PSM) and multicenter retrospective analysis to study the efficacy and toxicity of VMAT technology in radiotherapy for primary tumors of A-NSCLC.We used the chi-squared test to analyze the response rate(RR), local control (LC), acute radiation injury, and dose-volume parameters; the Kaplan-Meier and log-rank tests were used to determine local-regional progression-free survival (LRPFS) and overall survival (OS).

Results: LRPFS was significantly prolonged in stage III patients treated with IMRT before PSM $(P<.05)$ and cases of grade 1 or 2 acute radiation esophagitis (RE) or radiation pneumonia(RP) in the IMRT than the $\operatorname{VMAT}(P<.05)$, but cases of grade 3 or 4 RE and RP were not significantly different between the groups $(P>.05)$. Before PSM, there was no significant difference in the LRPFS and OS rates of the whole study group and the RR, LC, and stage IV subgroups, respectively ( $P>$.05). After PSM, there was no significant difference in the RR, LC, LRPFS, OS, RP, or RE of patients treated with VMAT and IMRT ( $P>$.05), normal whole-lung (V5, V20, MLD), heart (V30, V40, MHD), and equal dosevolume parameters were significantly lower in the VMAT group $(P<.05)$.

Conclusion:.Radiation therapy of A-NSCLC primary tumors using VMAT can achieve a similar efficacy to that of IMRT but with significantly lower low-dose volume parameters of normal tissues and organs; this is true especially in stage $\mathrm{N} 2$ cases, where the reduction in radiation damage may be more favorable.

\section{Introduction}

As three-dimensional radiotherapy is widely used in advanced non-small-cell lung cancer (A-NSCLC), prospective studies have shown that, based on chemotherapy, molecular targeted therapy combined with three-dimensional conformal radiotherapy (3D-CRT), intensity-modulated radiotherapy (IMRT), stereotactic body radiotherapy (SBRT), or stereotactic radiation ablation (SABR) can prolong overall survival (OS) [1-4], especially in patients with oligometastasis. Although two phase II prospective studies confirmed that randomization to 3D-CRT or IMRT for stage IV NSCLC prolonged survival at whole-lung V20 $\leq 32 \%$ and maximum spinal dose $\leq 50$ Gy, the characteristics of 3D-CRT or IMRT were not analyzed $[5,6]$. A phase II randomized controlled study of SBRT and drug-only treatment based on four cycles of chemotherapy or three months of molecular targeted therapy benefiting oligometastatic stage IV NSCLC patients recorded a significant prolongation of both progression-free survival (PFS) and overall survival (OS), but radiotherapy-only treatment involved irradiation of residual lesions and radiotherapy. The therapy plan was determined by the radiotherapists participating in the study, and there was no choice of radiotherapy technology or divided dose administration [7]. Another course of SABR or hyperfractionated radiotherapy for EGFRnegative stage IV NSCLC could also be used to irradiate the primary tumor or mediastinal metastatic lymph nodes. A randomized controlled phase II prospective study examining the benefit of chemotherapy alone or metastatic lesions also reported a significant prolongation of PFS; however, although it described the divided dose, this study did not mention the choice of three-dimensional radiotherapy technology to achieve SABR [8]. A meta-analysis of 21 studies confirmed that primary tumor radiotherapy significantly prolonged OS and PFS; among other results, it noted that radical dose radiotherapy may be more conducive to prolonging survival results. The studies included in the analysis used 3D-CRT, IMRT, SBRT, etc., and no radiotherapy was also used. The report included an analysis of technical characteristics [9]. T3-4 and N2-3 of A-NSCLC account for about $64 \%$ and $83 \%$, respectively. The primary tumor is of a late stage and large size, with spatial diversity [5], which are also factors that lead to the failure of first-line chemotherapy and molecular targeted therapy $[10,11]$. Therefore, the local control rate and survival are obtained to 
control radiation damage and improve tumor dose. In this study, volumetric modulated arc therapy (VMAT) was used to improve the conformity of radiotherapy for large-volume thoracic esophageal cancer and to reduce the V20 and dose (MU) of the whole lung [12]. In addition, in the external irradiation of cervical cancer, VMAT at an energy dose of 6-MV has the best conformation of two-arc therapy, the lowest bladder and rectal doses considering adjacent organs, and reliable radio-physical quality assurance $[13,14]$. The clinical value of VMAT in the treatment of primary tumors of A-NSCLC was retrospectively analyzed by pairing multicenter analysis and propensity score matching.

\section{Materials And Methods}

Criteria for case selection. Eligible patients had pathologically diagnosed and newly treated stage IIIA to IV (American Joint Committee on Cancer, eighth edition) NSCLC; were between 18 and 80 years old; had a Karnofsky performance status of at least 70; underwent primary tumor complete VMAT or IMRT radiotherapy with a radiation dose of at least 40Gy/20f; underwent chemotherapy for more than two cycles; received epidermal growth factor receptor EGFR (M+) first-line treatment with EGFR tyrosine kinase inhibitors; underwent complete recent results collection and acute radiotoxicity evaluations; and had complete follow-up data available. To determine the metastasis status, we defined oligometastasis $(\mathrm{OM})$ as one to five distance metastases $[15,16]$, while cases of at least six metastases were defined as non-OM (NOM).

Primary tumor radiotherapy. We first selected a 6-MV X-ray from Elekta Infinity linear accelerator then positioned patients in the supine position, performed thermoplastic film fixation, performed computed tomography simulation with a 5-mm-layer thickness enhancement scan to obtain the positioning image and transmit it to Pinnacle ${ }^{3}$ from Philips, and completed target acquisition according to the default lung window and mediastinal window of the planning system outline. The primary tumor (gross tumor volume [GTV]) is defined as the primary lesion and the metastatic lymph nodes in the drainage area. The metastatic lymph node must be a single lymph node with a short diameter of $\geq 1.0 \mathrm{~cm}$ or at least three lymph nodes in the same lymph drainage area with a diameter of $\geq 0.5 \mathrm{~cm}$. For this study, the clinical target area (clinical target volume [CTV]) was defined as a three-dimensional expansion of the GTV edge by $0.6 \mathrm{~cm}$ and combined with anatomical barriers, and the planning target volume (PTV) was defined as a three-dimensional expansion of the CTV edge by 0.5 to $1 \mathrm{~cm}$. The Pinnacle3 system was used to complete the radiotherapy plan design. IMRT of the primary tumor employed four to eight coplanar or non-coplanar fields, and VMAT required two to four arcs. The plan evaluation required that the prescribed dose cover $100 \%$ of the GTV volume; $95 \%$ of the prescribed dose for stage III cases included $95 \%$ or more of the PTV volume, and $90 \%$ of the prescribed dose for phase IV cases included $98 \%$ or more of the PTV volume. Considering a normal average dose of whole lung (total lung volume minus GTV) $\leq 20$ Gy and whole-lung (V20) dose $\leq 32 \%$,for primary tumor irradiation, we adopted late-course accelerated hyperfractionation (first course, 36-40Gy, 2Gy/time, 1 time/1 day, 5 days/week; latter course, 1.5Gy/time, 2 times/day, with an interval of 6 hours, 5 days/week).

Drug therapy. Chemotherapy involved a platinum-based two-drug combination regimen [17] of platinum drugs, and the dose regimen was as follows: cisplatin (DDP) $75 \mathrm{mg} / \mathrm{m}^{2}$ given as an intravenous infusion over two to four days or carboplatin (CBP) 300 to $350 \mathrm{mg} / \mathrm{m}^{2}$ given on day 2; paclitaxel (TAT) 135 to $175 \mathrm{mg} / \mathrm{m}^{2}$ given on day 1 or docetaxel (DOC) 60 to $75 \mathrm{mg} / \mathrm{m}^{2}$ given on day 1, with vinorelbine $30 \mathrm{mg} / \mathrm{m}^{2}$ also given on day 1; and pemetrexed (PEM) $500 \mathrm{mg} / \mathrm{m}^{2}$ given on day 1 (for cases of non-squamous cell carcinoma). A total of 21 to 28 days was considered one cycle, with two to six cycles performed in a row.

Molecular targeted therapy employs first-line drugs based on the results of sensitive mutations in driving genes, with gefitinib $250 \mathrm{mg}$ taken orally every day, icotinib $150 \mathrm{mg}$ taken orally three times a day, osimertinib $80 \mathrm{mg}$ taken orally

Page $4 / 14$ 
every day, and crizotinib $250 \mathrm{mg}$ taken orally twice a day, until the disease progresses.

Evaluation of the recent results for primary tumors. According to the RECIST 1.1 standard [18], the efficacy evaluation allocated patients into complete response (CR), partial response (PR), stable disease (SD), and progressive disease (PD) groups; for this study, CR+PR was defined as effective (response rate [RR]), and a result of CR+PR+SD suggested the disease to be controlled (disease control rate $[D C R])$. The effective rate $=(C R+P R) /$ total number of cases $\times 100 \%$, while the disease control rate $=(C R+P R+S D) /$ total number of cases $\times 100 \%$.

Acute radiation injury classification. According to the radiation injury classification standards of the Radiotherapy Oncology Group (RTOG), acute injuries to the lungs, esophagus, and/or heart were recorded.

Observation endpoints. The main observation endpoints were the recent results of primary tumor analysis, local control rate (LCR), local-regional PFS (LRPFS), and acute radiation pneumonia (radiation pneumonia [RP]) and esophagitis (radiation esophagitis [RE]). The secondary observation endpoints are the whole-lung (V5, V20, MLD), heart (V30, V40), esophagus (V50), and other dose-volume parameters as well as two-year survival (OS).

Statistical method. Using the Statistical Package for the Social Sciences version 26.0 software program (IBM Corporation, Armonk, NY, USA), characteristics including sex, age, pathological type, T/N/M staging, GTV volume, primary lesion location, clinical stage, metastasis status, targeted therapy, chemotherapy cycle, prescription dose, and other factors for IMRT and PSM of VMAT showed a matching tolerance of 0.02. Survival analysis was tested using the Kaplan-Meier and log-rank methods; local control rate was calculated by the life table method; and recent measurements, radiation injury, and dose-volume parameters were tested using the chi-squared test. A $P$-value of .05 or less was considered statistically significant.

\section{Results}

Clinical characteristics of the case data. From September 2008 to March 2020, 637 cases met the case selection criteria. In the study population, the male to female ratio was $2.5: 1$; the median age was 58 years (range, 22-79 years); and there were 51, 91, 68, and 427 cases of stages IIIA, IIIB, IIIC, and IV cancer, respectively. A total of 102 cases of driver gene-sensitive mutations were detected before treatment due to economic and medical insurance factors (including 83 cases of EGFR mutations and 19 cases of $A L K$ mutations), and only $10.20 \%$ of patients received molecular targeted therapy (including gefitinib in 28 cases, icotinib in 22 cases, ositinib in two cases, and crizotinib in 13 cases), while chemotherapy was used in $89.80 \%$ of cases. The proportions of N2-3 and IV stages among IMRT patients before PSM was higher than those among VMAT $(P<.05)$, and the prescribed dose and GTV volume were similar $(P>$.05). After PSM, there were 308 patients (154 pairs) with a median age of 58 years. The clinical baseline conditions of the two groups were similar $(P>.05)$. See Table 1 for details. 
Table 1

Clinical baseline characteristics of 637 patients with advanced NSCLC treated with IMRT or VMAT before and after PSM

\begin{tabular}{|c|c|c|c|c|c|c|}
\hline & Before PS & & & After PSN & & \\
\hline Variable & $\begin{array}{l}\text { IMRT } \\
(n=483)\end{array}$ & $\begin{array}{l}\text { VMAT } \\
(n=154)\end{array}$ & $P$-value & $\begin{array}{l}\text { IMRT } \\
(n=154)\end{array}$ & $\begin{array}{l}\text { VMAT } \\
(n=154)\end{array}$ & $P$-value \\
\hline Sex (Male/female) & $344 / 139$ & $113 / 41$ & .606 & $113 / 41$ & $113 / 41$ & 1.000 \\
\hline Age (years) & $22-79$ & $28-78$ & .717 & $30-77$ & $28-78$ & .680 \\
\hline Median age (years) & 58 & 58 & & 58 & 58 & \\
\hline Pathological type & & & .179 & & & .426 \\
\hline Squamous cell carcinoma & 180 & 64 & & 73 & 64 & \\
\hline Adenocarcinoma & 273 & 84 & & 74 & 84 & \\
\hline Other & 30 & 6 & & 7 & 6 & \\
\hline Primary lesion site & & & .404 & & & .458 \\
\hline Right upper lung & 125 & 43 & & 48 & 43 & \\
\hline Right middle lung & 50 & 12 & & 17 & 12 & \\
\hline Right lower lung & 87 & 39 & & 29 & 39 & \\
\hline Upper left lung & 129 & 34 & & 38 & 34 & \\
\hline Lower left lung & 92 & 26 & & 22 & 26 & \\
\hline Gene mutation & 54 & 48 & .857 & 47 & 48 & .727 \\
\hline Targeted therapy & 50 & 15 & .827 & 20 & 15 & .547 \\
\hline Chemotherapy & 433 & 139 & .260 & 134 & 139 & .827 \\
\hline Transfer situation & & & .058 & & & .831 \\
\hline Oligotransfer & 302 & 63 & & 69 & 63 & \\
\hline Non-oligo transfer & 45 & 17 & & 19 & 17 & \\
\hline T stage & & & .998 & & & .324 \\
\hline $\mathrm{T} 1-2$ & 160 & 51 & & 43 & 51 & \\
\hline T3-4 & 323 & 103 & & 111 & 103 & \\
\hline $\mathrm{N}$ stage & & & .035 & & & .874 \\
\hline No-1 & 61 & 10 & & 10 & 10 & \\
\hline N2 & 157 & 53 & & 51 & 53 & \\
\hline N3 & 265 & 91 & & 93 & 91 & \\
\hline M stage & & & .000 & & & .437 \\
\hline
\end{tabular}




\begin{tabular}{|c|c|c|c|c|c|c|}
\hline & Before PSM & & & After PSM & & \\
\hline MO & 136 & 74 & & 66 & 74 & \\
\hline M1a & 57 & 20 & & 17 & 20 & \\
\hline M1b & 203 & 23 & & 36 & 23 & \\
\hline M1c & 87 & 37 & & 35 & 37 & \\
\hline Stage (III/IV) & $136 / 347$ & $74 / 80$ & .000 & $66 / 88$ & $74 / 80$ & .491 \\
\hline $\operatorname{GTV}\left(\mathrm{cm}^{3}\right)$ & & & .335 & & & .104 \\
\hline III & $205 \pm 185$ & $212 \pm 193$ & & $216 \pm 190$ & $212 \pm 193$ & \\
\hline IV & $224 \pm 179$ & $194 \pm 169$ & & $239 \pm 151$ & $194 \pm 169$ & \\
\hline III+IV & $216 \pm 181$ & $200 \pm 175$ & & $228 \pm 167$ & $200 \pm 175$ & \\
\hline Prescribed dose/median (Gy) & $40-76.5 / 63$ & $40-71 / 64$ & .056 & $40-76 / 63$ & $40-71 / 64$ & .190 \\
\hline
\end{tabular}

Recent efficacy of radiotherapy with different techniques before and after PSM for primary tumors. The analysis of recent efficacy of treatment with IMRT and VMAT showed that cases of CR, PR, SD, and PD following IMRT and VMAT before PSM were $1.7 \%, 69.4 \%, 18.2 \%$, and $10.8 \%$ and $1.3 \%, 72.1 \%, 21.4 \%$, and $5.2 \%$, respectively. The RR $\left(X^{2}=\right.$ 1.037; $P=.309)$ and DCR $\left(X^{2}=2.781 ; P=.427\right)$ of the two technologies had no significant differences. After PSM, cases of CR, PR, SD, and PD in the IMRT and VMAT groups were 1.3\%, 72.7\%, 19.5\%, 6.5\%, and 1.3\%, 72.1\%, $21.4 \%$, and $5.2 \%$, respectively. The RR $\left(\chi^{2}=1.662 ; P=.197\right)$ and $\operatorname{DCR}\left(\chi^{2}=0.370 ; P=.946\right)$ of the two technologies had no significant differences. See Table 2.

Table 2

The recent results of IMRT and VMAT before and after PSM in the treatment of primary tumors

\begin{tabular}{|c|c|c|c|c|c|c|c|c|c|c|c|c|}
\hline & Technology & Cases & CR & PR & SD & PD & $\begin{array}{l}\text { RR } \\
(\%)\end{array}$ & $x^{2}$ & $\begin{array}{l}P \\
\text { value }\end{array}$ & $\begin{array}{l}\text { DCR } \\
\text { (\%) }\end{array}$ & $\chi^{2}$ & $\begin{array}{l}P \\
\text { value }\end{array}$ \\
\hline \multirow{2}{*}{$\begin{array}{l}\text { Before } \\
\text { PSM }\end{array}$} & IMRT & 483 & 8 & 335 & 88 & 52 & 71.01 & \multirow[t]{2}{*}{1.037} & \multirow[t]{2}{*}{0.309} & 89.23 & \multirow[t]{2}{*}{2.781} & \multirow[t]{2}{*}{.427} \\
\hline & VMAT & 154 & 2 & 111 & 33 & 8 & 73.37 & & & $94.8 \%$ & & \\
\hline After & IMRT & 154 & 2 & 112 & 30 & 10 & 72.72 & \multirow[t]{2}{*}{1.662} & \multirow[t]{2}{*}{0.197} & $93.50 \%$ & \multirow[t]{2}{*}{0.370} & \multirow[t]{2}{*}{.946} \\
\hline PSM & VMAT & 154 & 2 & 111 & 33 & 8 & 73.37 & & & $94.80 \%$ & & \\
\hline
\end{tabular}

LCR of primary tumors before and after PSM with different techniques for radiotherapy. Before PSM, local control rates at one and two years after treatment with IMRT ( $n=483$ cases) or VMAT ( $n=154$ cases) were $93.2 \%$ and $93.3 \%$ and $76.2 \%$ and $86.1 \%$,respectively, with no statistically significant differences $\left(\chi^{2}=0.292 ; P=.589\right)$. There was also no statistically significant difference between $93.50 \%$ and $93.3 \%$ and $76.1 \%$ and $86.1 \%$, respectively. $\left(\chi^{2}=0.467 ; P=\right.$ $.490)$.

LRPFS of different techniques for radiotherapy of primary tumors before and after PSM. Before PSM, the one-year and two-year LRPFS rates following treatment with IMRT or VMAT were $91.1 \%$ and $91.9 \%$ and $76.0 \%$ and $86.1 \%$, respectively, and there was no statistically significant difference $\left(\chi^{2}=0.246 ; P=.620\right)$. The stratified analysis showed that the LRPFS of stage III at one and two years were $97.6 \%$ and $90.6 \%$ and $91.9 \%$ and $83.0 \%$, respectively, and the 
LRPFS of IMRT treatment was prolonged $\left(\chi^{2}=4.183 ; P=.041\right)$. The one-year and two-year LRPFS of stage IV were $91.0 \%$ and $93.2 \%$ and $67.8 \%$ and $88.7 \%$, respectively, with no statistically significant difference $\left(\chi^{2}=2.097 ; P=.148\right)$. See Figure $1(\mathrm{a}-\mathrm{c})$ for details. After PSM, the one-year and two-year LRPFS rates among all patients treated with IMRT or VMAT were $92.1 \%$ and $91.9 \%$ and $76.1 \%$ and $86.1 \%$, respectively $\left(\chi^{2}=0.473 ; P=.492\right)$. Among the stage III cases, the one-year and two-year LRPFS rates were $94.1 \%$ and $90.6 \%$ and $88.5 \%$ and $83.0 \%\left(\chi^{2}=1.697 ; P=.193\right)$. Among the stage IV cases, the one-year and two-year LRPFS rates were $88.7 \%$ and $93.2 \%$ and $64.9 \%$ and $88.7 \%$, respectively $\left(\chi^{2}=3.132 ; P=.077\right)$, and there was no statistically significant difference. See Figure $2(a-c)$.

The one- and two-year OS of radiotherapy with different techniques after PSM. The one-year and two-year OS rates after IMRT or VMAT treatment were $70.1 \%$ and $69.9 \%$ and $31.3 \%$ and $50.1 \%$, respectively $\left(X^{2}=1.504 ; P=.220\right)$, and there was no statistically significant difference. Stratified analysis showed that the one-year and two-year OS rates of stage III patients were $81.8 \%$ and $71.6 \%$ and $44.7 \%$ and $67.1 \%$, respectively $\left(\chi^{2}=0.022 ; P=.881\right)$, and there was no statistically significant difference. Finally, the one-year and two-year OS rates of stage IV patients were $67.3 \%$ and $61.3 \%$ and $21.4 \%$ and $37.6 \%$, respectively $\left(X^{2}=2.207 ; P=.137\right)$, and there was no statistically significant difference. See Figure $3(a-c)$ for details.

$R P$ and RE of different radiotherapy techniques before and after PSM. There was no grade VI injury in the whole study group. The incidence rates of RE and RP in the IMRT group before PSM were $73.70 \%$ and $36.02 \%$, respectively, which were significantly higher than those of the VMAT group $(P<.05)$, The incidence rates of grade III or IV RE and RP were $8.28 \%$ and $7.14 \%$ and $7.03 \%$ and $7.79 \%$ for IMRT and VMAT, respectively, with no statistically significant difference $\left(\chi^{2}=2.77 ; P=.096\right)$. There was also no significant difference after PSM. See Table 3 for details.

Table 3

IMRT and VMAT radiotherapy before and after PSM for acute radiation injury of primary tumors

\begin{tabular}{|c|c|c|c|c|c|c|c|c|c|c|c|c|c|}
\hline \multirow[t]{2}{*}{ Group } & \multirow{2}{*}{$\begin{array}{l}\text { Radiation } \\
\text { damage }\end{array}$} & \multicolumn{5}{|c|}{ IMRT } & \multicolumn{5}{|c|}{ VMAT } & \multirow{2}{*}{$\begin{array}{l}\chi^{2} \\
\text { value }\end{array}$} & \multirow{2}{*}{$\begin{array}{l}P \text { - } \\
\text { value }\end{array}$} \\
\hline & & 0 & I & II & III & IV & 0 & I & II & III & IV & & \\
\hline Before & $\mathrm{RE}$ & 127 & 172 & 144 & 40 & 0 & 61 & 54 & 28 & 11 & 0 & 13.04 & .005 \\
\hline PSM & $\mathrm{RP}$ & 309 & 110 & 30 & 33 & 1 & 115 & 17 & 10 & 12 & 0 & 10.75 & .029 \\
\hline After & RE & 60 & 50 & 26 & 18 & 0 & 61 & 54 & 28 & 11 & 0 & 1.336 & .721 \\
\hline PSM & RP & 109 & 21 & 14 & 10 & 0 & 115 & 17 & 10 & 12 & 0 & 0.533 & .912 \\
\hline
\end{tabular}

IMRT and VMAT dose-volume parameters after PSM. N2 stage IMRT whole-lung V5, V20, MLD, heart V30, V40, MHD, and esophagus V50 were significantly higher than in the VMAT group $(P<.05)$; further, N3 stage IMRT whole-lung V5 was higher than that in the VMAT group $(P<.05)$. There was no significant difference in esophageal V50 of the whole group of patients $(P>.05)$. See Table 4 for details. 
Table 4

Normal tissue dose-volume parameters of IMRT and VMAT radiotherapy for primary tumor after PSM

\begin{tabular}{|c|c|c|c|c|}
\hline Variable & Staging & IMRT & VMAT & $P$ value \\
\hline \multirow[t]{3}{*}{ All lungV5 } & All patients & $67.06 \pm 13.36$ & $56.90 \pm 16.28$ & .000 \\
\hline & N2 & $63.74 \pm 14.41$ & $48.22 \pm 15.84$ & .000 \\
\hline & N3 & $70.55 \pm 13.66$ & $62.50 \pm 13.72$ & .000 \\
\hline \multirow[t]{3}{*}{ All lungV20 } & All patients & $28.10 \pm 6.17$ & $25.71 \pm 6.88$ & .001 \\
\hline & N2 & $27.02 \pm 6.30$ & $22.36 \pm 7.76$ & .000 \\
\hline & N3 & $29.29 \pm 4.95$ & $28.39 \pm 4.84$ & .154 \\
\hline \multirow[t]{3}{*}{ MLD } & All patients & $17.82 \pm 3.54$ & $16.21 \pm 4.34$ & .001 \\
\hline & N2 & $17.30 \pm 4.18$ & $14.30 \pm 4.95$ & .000 \\
\hline & N3 & $18.30 \pm 3.27$ & $17.88 \pm 3.20$ & .312 \\
\hline Heart & All patients & $25.36 \pm 12.88$ & $21.58 \pm 11.80$ & .010 \\
\hline \multirow[t]{2}{*}{ V30 } & N2 & $25.29 \pm 13.44$ & $17.04 \pm 11.91$ & .000 \\
\hline & N3 & $26.99 \pm 14.73$ & $24.89 \pm 10.82$ & .230 \\
\hline Heart & All patients & $17.09 \pm 9.77$ & $14.71 \pm 8.99$ & .017 \\
\hline \multirow[t]{2}{*}{ V40 } & N2 & $17.52 \pm 10.85$ & $11.98 \pm 8.41$ & .001 \\
\hline & N3 & $17.82 \pm 10.91$ & $16.85 \pm 8.90$ & .463 \\
\hline \multirow[t]{3}{*}{ MHD } & All patients & $19.77 \pm 8.65$ & $17.22 \pm 8.09$ & .019 \\
\hline & N2 & $19.63 \pm 8.62$ & $13.74 \pm 8.23$ & .000 \\
\hline & N3 & $20.36 \pm 8.77$ & $19.66 \pm 7.38$ & .515 \\
\hline Esophagus & All patients & $32.95 \pm 20.03$ & $32.37 \pm 19.99$ & .800 \\
\hline \multirow[t]{2}{*}{ V50 } & N2 & $34.84 \pm 18.33$ & $28.60 \pm 18.02$ & .046 \\
\hline & N3 & $36.80 \pm 20.05$ & $35.22 \pm 21.05$ & .545 \\
\hline
\end{tabular}

\section{Discussion}

The results of this study show that the male:female ratio is $2.5: 1$, the median age is 58 years, and that adenocarcinoma and squamous cell carcinoma cases total about $94.3 \%$ of the study population, which is similar to the epidemiological characteristics of the incidence of NSCLC [19]. Also, T3-4 and N2-3 cases totaled 67\% and $88.9 \%$, respectively. The average volume of GTV was about $200 \mathrm{~cm}^{3}$. There was no significant difference in the distribution of primary lesions between patients who received VMAT and IMRT, respectively. The disease stage was late, and mediastinal lymph node metastasis was obvious [20,5]. The proportions of N2-3 and IV patients, respectively, in the pre-PSM IMRT group were relatively high, regardless of stage III or oligometastasis of more than $50 \%$ among stage IV NSCLC patients. Both results show that the primary tumor is large in size [21], and it is difficult to obtain the local control rate by increasing the dose under the premise of controlling the damage. It is necessary to 
use the physical characteristics of different three-dimensional radiotherapy techniques to explore the clinical application value.

The difference between VMAT and IMRT as treatment is that, during the accelerator irradiation process, the rotating irradiation is realized by continuous changes of the gantry speed, collimator angle, and dose rate, and it has the characteristic of a short irradiation time. For elderly patients older than 80 years old with irregular target areas and more protected organs at risk, studies on head and neck tumors have shown that VMAT can ensure target dose and improve conformability while significantly reducing the exposure to endangered organs so as to obtain the same tumor-free survival rate as younger patients. Considering the tumor-free survival rate [22], a small A-NSCLC radiation therapy plan dose-volume parameter study showed that VMAT increased the V95\% and conformity of the planned target area and reduced the average dose to the lungs, esophagus, and heart [23] compared to IMRT. Therefore, in this study, our multicenter and retrospective analysis of VMAT treatment results through the trend score showed that the RR, DCR, and LCR of PSM before and after IMRT treatment were similar $(P>.05)$, suggesting that the volume and primary tumor dose are similar. Under this premise, application of VMAT in A-NSCLC patients is effective in the treatment of primary tumors, and the recent results were not less than those of IMRT. Although it was shown before PSM that the LRPFS of stage III NSCLC cases treated with IMRT was longer than that of VMAT, there was no significant difference in LRPFS in the whole group nor during stratified analysis under the premise of the same baseline conditions after PSM, indicating that VMAT combined with drugs can be used in the first-line treatment of ANSCLC with a long-term efficacy similar to that of IMRT. Moreover, the one-and two-year OS rates of VMAT combined with chemotherapy in the first-line treatment of stage IV NSCLC patients were $61.3 \%$ and $37.6 \%$, respectively, which may be much higher than the one- and two-year OS rates of $35 \%$ and $10 \%$, respectively, associated with platinumcontaining two-drug regimen first-line chemotherapy [24], suggesting a prolonged survival role [4]. In summary, the effect of VMAT in the treatment of A-NSCLC is similar to that of IMRT. It improves recent results and improves OS. The proportion of primary tumor volume shrinkage is also negatively correlated with the risk of progression failure, and OS is prolonged through radiotherapy, as well as an increased dose and local control rate [5, 25].

It is well known that acute radiation injury is the dose-limiting toxicity of radiotherapy, RE and RP of grade 3 or higher are unfavorable factors for the prognosis of NSCLC [26], and the volume of normal lung low-dose radiation is related to the occurrence of RP. VMAT irradiation needs to be performed through a rotating arc of the gantry, but the low dose volume of normal tissues does not necessarily increase. This study showed that the incidence rates of RP and RE of primary A-NSCLC tumors with a median dose of 64Gy and an average volume of more than $200 \mathrm{~cm}^{3}$ after VMAT treatment were lower than that of IMRT before PSM. There was no significant difference in grade 3 and 4 acute radiation injury between groups, and both results were determined after PSM. The absence of a significant difference suggests that the rotating irradiation mode of VMAT did not increase the acute radiation injury caused by the low dose volume of normal tissue in clinical practice. In addition, this study analyzed the MHD isometric volume parameters of the heart in the dose-volume histogram as well as the important indicators for predicting the occurrence of RE and RP; esophagus V50; and whole-lung V5, V20, and MLD [27-31]. After PSM, the cardiac dosevolume parameters of patients treated with VMAT and the low dose volume of normal lungs were significantly lower than those of the IMRT group. In particular, the stratified analysis showed that the dose parameters, such as MHD, esophagus V50, and normal whole-lung V5 of N2 staging cases were significantly lower than those of the IMRT. The IMRT group was significantly reduced $(P<.05)$, while N3 patients with only normal whole-lung V5 had parameters significantly lower than those of the IMRT group $(P=.000)$, further suggesting that the VMAT rotary irradiation method has a more reasonable dose distribution and maintains the low dose volume to reduce radiation damage. The VMAT rotary irradiation method has the advantages of a more reasonable dose distribution and better control of low dose volume to reduce radiation damage, especially in patients with mediastinal lymph node metastasis only on 
the same side. More importantly, the reduction in dose volume may reduce the late damage of normal tissues, especially considering the reduction of heart damage that is positively correlated with the radiation dose and clinical dysfunction that occurs for several years or longer after irradiation [32-33].

\section{Conclusions}

The selection of VMAT for primary tumor radiotherapy in A-NSCLC patients can lead to the same recent and longterm efficacy results as IMRT, and it may be more advantageous to use this approach to reduce acute radiation injury, especially for late cardiac injury.

\section{Declarations}

\section{Author's contributions}

$J L$ prepared the manuscript, conducted the literature search, and reviewed and edited the manuscript. TL, WXH, SFS,QSL YCG, WGY, XXC, WWOY,WZand LB reviewed the manuscript. All authors have read and approved the final manuscript.

\section{Funding}

Guizhou Provincial Department of Science and Technology Project

Contract No. Qianke He Support [2019] No. 2795.

\section{Availability of data and materials}

The data that support the findings of this study are available on request from the corresponding author.

Code availability.

Not applicable.

\section{Ethics approval and consent to participate}

This retrospective analysis was approved by the ethics committee of the ethics committee of the Affiliated Hospital of Guizhou Medical University Ethics approval document, (approval number:2021-275). Informed consent was obtained from all individual participants included in the study.

\section{Consent for publication}

Informed consent was obtained from all individual participants included in the study.

\section{Competing interests}

Jie Liu, Tao Li,Wang Xiaohu , Shengfa Su, Qingsong Li, Yichao Geng, Wengang Yang, Xiaxia Chen,Weiwei Ouyang,Wei Zhang and Bing Lu declare that they have no competing interests.

\section{Author details}

${ }^{1}$ Guizhou Medical University 
2 Sichuan Cancer Hospital and Institute

${ }^{3}$ The First Clinical Medical College of Lanzhou University;Gansu Provincial Cancer Hospital

${ }^{4}$ Affiliated Hospital of Guizhou Medical University, and Guizhou Cancer Hospital.

\section{References}

1. Kunitoh $\mathrm{H}$, Kato $\mathrm{H}$, Tsuboi $\mathrm{M}$, et al. Phase II trial of preoperative chemoradiotherapy followed by surgical resection in patients with superior sulcus non-small-cell lung cancers: report of Japan Clinical Oncology Group trial 9806.[J].Journal of Clinical Oncology, 2008, 26(4):644-649.

2. Blake-Cerda M, Ruiz FL, Magos FM,et al.Stereotactic ablative radiation therapy to lung metastases associates with better outcomes in oligometastatic lung cancer: prospective study[J]. J Thorac Oncol, 2019, 14(10):S766S767.

3. Zheng L, Wang Y, Xu Z,et al. Concurrent EGFR-TKI and Thoracic Radiotherapy as First-Line Treatment for Stage IV Non-Small Cell Lung Cancer Harboring EGFR Active Mutations[J]. Oncologist, 2019, 24(8):1031-1112.

4. Su S F,Hu Y X,Ouyang W W, et al.The survival outcomes and prognosis of stage IV non-small-cell lung cancer treated with thoracic three-dimensional radiotherapy combined with chemotherapy[J].Radiation Oncology,2014,9(1):1-7.

5. Su SF,Hu YX,Ouyang WW,et al.Overall survival and toxicities regarding thoracic three-dimensional radiotherapy with concurrent chemotherapy for stage IV non-small cell lung cancer results of a prospective single-center study J.BMC Cancer 2013,13,465-474.

6. Su S,Li T,Lu B,et al.Three-dimensional radiation therapy to the primary tumor with concurrent chemotherapy in patients with stage iv non-small cell lung cancer results of a multicenter phase 2 study from PPRA-RTOG China [J]. Int J Radiat Oncol Biol Phys 2015,93,4:769-777.

7. Gomez D R, Blumenschein G R,Lee J J, et al.Local consolidative therapy versus maintenance therapy or observation for patients with oligometastatic non-small-cell lung cancer without progression after first-line systemic therapy:a multicentre, randomised, controlled, phase 2 study[J]. Lancet Oncology, 2016, 17(12):16721682.

8. Puneeth lyengar,et al.Consolidative Radiotherapy for Limited Metastatic Non-Small-Cell Lung CancerA Phase 2 Randomized Clinical Trial[J]. JAMA Oncology, 2018, 4(1):7-30.

9. Petrelli F, Ghidini A, Cabiddu M, et al. Addition of Radiotherapy to the Primary Tumour in Oligometastatic NSCLC: A Systematic Review and Meta-Analysis[J]. Lung Cancer, 2018, 126:194-200.

10. Mehta N, Mauer A M, Hellman S, et al. Analysis of further disease progression in metastatic non-small cell lung cancer: implications for locoregional treatment.[J]. International Journal of Oncology, 2004, 25(6):1677-1683.

11. Wang B, Xin Z, Lin L, et al. Progressive patterns of gifitinib treating advanced non-small cell lung cancer after obtained resistance.[J]. Chinese Journal of Lung Cancer, 2013, 16(10):510-513.

12. Ahmar S, Abbas, et al. Volumetric-modulated arc therapy for the treatment of a large planning target volume in thoracic esophageal cancer[J]. Journal of Applied Clinical Medical Physics, 2013, 14(3):192-202

13. YADAV G,BHUSHAN M,DEWAN A, et al. Dosimetric influence of photon beam energy and number of arcs on volumetric modulated arc therapy in carcinoma cervix:a planning study[J].Rep Pract OncolRadiother,2017,22(1):1-9. 
14. DURSUN P,TASKIN Z C,ALTINEL I K. Mathematical models for optimal volumetric modulated arc therapy (VMAT) treatment planning[C].Procedia Computer Science,2016,100:644-651.

15. Kang X,Chen K.The Conceptual Oligometastatic Non-small Cell Lung Cancer and Therapeutic Strategies[J].Chinese Journal of Lung Cancer,2012.Chin J Lung Cancer,2012, 15,(4):242-245

16. RUSTHOVEN CG,YEH N,GASPAR L E.Radiation Therapy for Oligometastatic Non-Small Cell Lung Cancer:Theory and Practice[J],Cancer journal(Sudbxory,Mass), 2015,21(5):404-412

17. Delbaldo C,Michiels S,Syz N,et al.Benefits of adding a drug to a single-agent or a 2-agent chemotherapy regimen in advanced non-small-cell lung cancer:a meta-analysis [J].Jama,2004,292(4):470-484.

18. Eisenhauer E A, Therasse P, Bogaerts J, et al. New response evaluation criteria in solid tumours: revised RECIST guideline (version 1.1).[J]. European Journal of Cancer, 2009, 45(2):0-247.

19. Ferlay Jacques, Colombet Murielle, Soerjomataram Isabelle, Parkin Donald M.Piñeros Marion, Znaor Ariana, Bray Freddie. Cancer statistics for the year 2020:An overview[J]. International Journal of Cancer,2021,149(4):778789.

20. Morgensztern D,Ng S H,Feng G,et al. Trends in Stage Distribution for Patients with Non-small Cell Lung Cancer[J]. Journal of thoracic oncology:official publication of the International Association for the Study of Lung Cancer, 2009, 5(1):29-33.

21. Yang Yu,Hui Guan,Li-Gang Xing,Yong-Bing Xiang. Role of Gross Tumor Volume in the Prognosis of Non-small Cell Lung Cancer Treated With 3D Conformal Radiotherapy: A Meta-analysis[J]. Clinical Therapeutics,2015,37(10):1-11

22. Brown M L, Glanzmann C, Huber G, et al. IMRT/VMAT for malignancies in the head-and-neck region[J]. Strahlentherapie und Onkologie, 2016, 192(8):526-536.

23. Della GG, MLP D, Hoekstra N, et al.Fully automated VMAT treatment planning for advanced-stage NSCLC patients [J].Strahlenther Onkol, 2017,193 (5):402-409.

24. MD Fisher,D'Orazio A.Phase II and III Trials: Comparison of Four Chemotherapy Regimens in Advanced NonSmall-Cell Lung Cancer (ECOG 1594)[J]. Clinical Lung Cancer, 2001, 2(1):21-22.

25. Kong $\mathrm{F} M$, Haken $\mathrm{R}$, Schipper $\mathrm{M}$ J, et al.High-dose radiation improved local tumor control and overall survival in patients with inoperable/unresectable non-small-cell lung cancer: long-term results of a radiation dose escalation study.[J]. International Journal of Radiation Oncology Biology Physics, 2005, 63(2):324-333.

26. Palma DA,Senan S,Tsujino K,et al.Predicting radiation pneumonitis after chemoradiation therapy for lung cancer: an international individual patient data meta -analysis[J]. Int J Radiat Oncol Biol Phys,2013,85(2):444-450.

27. Giuliani ME, Lindsay PE, Kwan JY, et al. Correlation of dosimetric and clinical factors with the development of esophagitis and radiation pneumonitis in patients withlimited $\llbracket$ stage small『cell lung carcinoma[J]. Clin Lung Cancer,2015,16(3):216ख220.

28. Konski A, Doss M, Milestone B, et al. The integration of 18®fluorodeoxyglucose positron emission tomography and endoscopic ultrasound in the treatment $₫$ planning process for esophageal carcinoma[J].Int J Radiat Oncol Biol Phys, 2005,61(4): 1123囚1128.

29. Muijs CT, Schreurs LM, Busz DM, et al. Consequences of additional use of PET information for target volume delineation and radiotherapy dose distribution for esophageal cancer[J]. Radiother Oncol, 2009, 93(3): $447 \rrbracket 453$.

30. Hill R P, Bristow R G, Fyles A, et al. Hypoxia and Predicting Radiation Response[J]. Seminars in Radiation Oncology, 2015,25(4):260-272.

31. Jumeau R, É Filion, Bahig H, et al. A dosimetric parameter to limit chest wall toxicity in SABR of NSCLC[J]. British Journal of Radiology, 2017,90(7):1-14.

Page $13 / 14$ 
32. Frandsen J, D Boothe, Gaffney D K, et al. Increased risk of death due to heart disease after radiotherapy for esophageal cancer[J]. Journal of Gastrointestinal Oncology, 2015, 6(5):516-23.

33. Umezawa R, Kadoya N, H Ota, et al. Dose-dependent Radiation-induced Myocardial Damage in Esophageal Cancer Treated with Chemoradiotherapy:A Prospective Cardiac Magnetic Resonance Imaging Study[J]. Advances in Radiation Oncology, 2020.5(6):1170-1178.

\section{Figures}
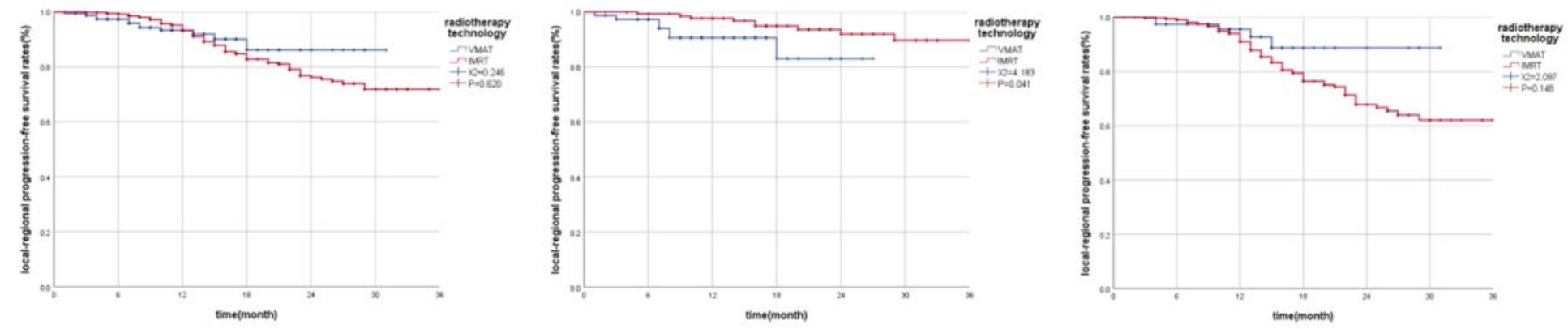

Figure 1

IMRT and VMAT radiotherapy for primary tumor LRPFS before PSM. (a) Stages III through IV, (b) stage III, and (c) stage IV.
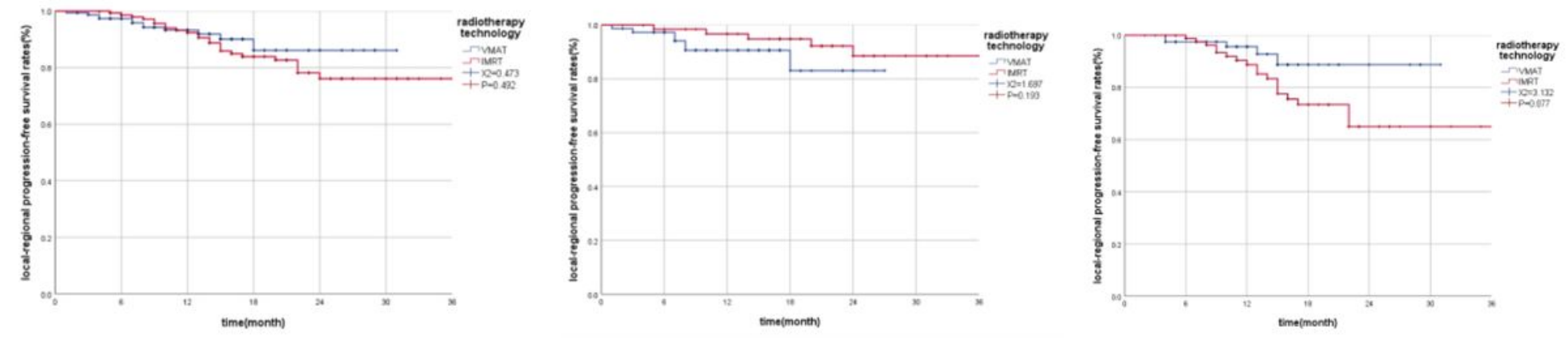

\section{Figure 2}

IMRT and VMAT radiotherapy for primary tumor LRPFS after PSM. (a) Stages III through IV, (b) stage III, and (c) stage IV.
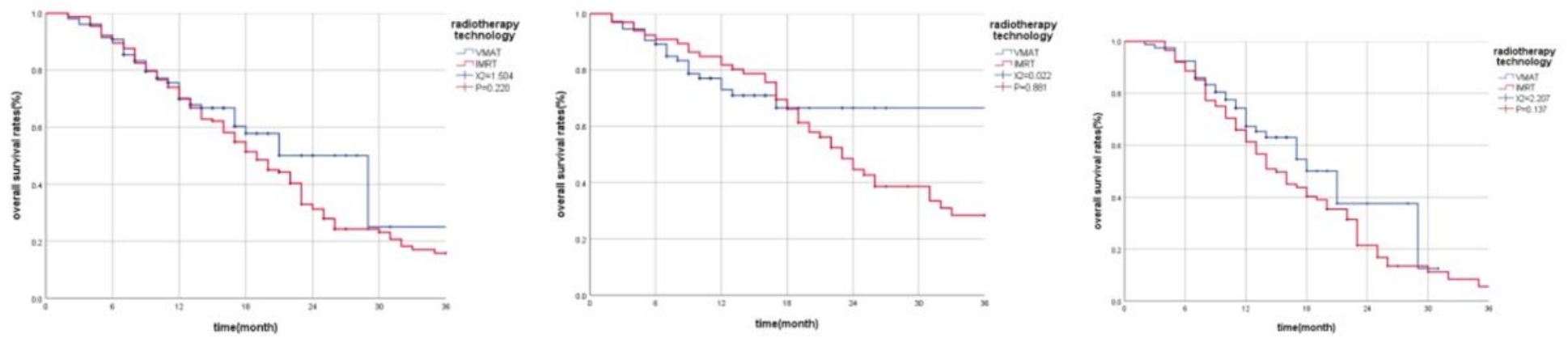

\section{Figure 3}

The OS of IMRT and VMAT primary tumor radiotherapy after PSM. (a) Whole group, (b) stage III, and (c) stage IV. 